\title{
Triple hydrogen-bonding containing materials: RAFT polymerization in the presence of 1-octylthymine and self-assembly behavior
}

\author{
Shixue Wang, Youhua Tao* and Xianhong Wang
}

\begin{abstract}
The synthesis of triple hydrogen-bonding block copolymers by sequential reversible addition-fragmentation chain transfer (RAFT) polymerizations was explored. In combination with 1-octylthymine as mediator, the amphiphilic triple hydrogen-bonding block copolymers with $M_{\mathrm{n}}$ up to 24,900 Da and relatively broader molecular weight distribution were obtained. Micelles with hydrophobic core and hydrophilic corona could be fabricated by self-assembling of the corresponding block copolymer in water. Transmission electron microscopy and laser light scattering studies show that the micelles are in nano-scale with narrow size distribution. The obtained polymeric micelle with triple hydrogen-bonding unit could be used in biomedical materials field such as carrier of hydrophilic drug.
\end{abstract}

\section{INTRODUCTION}

Among the various non-covalent interactions, the multiple hydrogen-bonding interactions are quite strong [1-3]. Therefore, multiple hydrogen-bonding interactions have been used for building supramolecular polymers and driving self-assembly [4-7]. Moreover, multiple hydrogen-bonding interactions between the designed arrays of the proton-donating (D) and accepting (A) sites, have been employed in synthetic polymer chemistry to mediate polymer composition or sequence and to template polymerizations [8-14]. These pioneering works have expanded the scope of further investigation of the multiple hydrogen-bonding materials.

The multiple hydrogen-bonding polymers can be prepared by direct polymerization of styrenic and methacrylic monomers containing multiple hydrogen-bonding unit [15]. Various polymerization methods are used to synthesize multiple hydrogen-bonding polymers, including ring-opening metathesis polymerization [16-20], atom transfer radical polymerization (ATRP) and reversible addition-fragmentation chain transfer (RAFT) polymerization [8,14,21-24]. Lutz et al. reported the synthesis of styrenic polymers with multiple hydrogen-bonding sites and observed the association and dissociation of hydrogen bonding in solution [25]. Long's group demonstrated the synthesis of styrenic triblock copolymers bearing pendant multiple hydrogen-bonding units and showed their potential as drug delivery vehicles and biological probes [26]. Recently, we synthesized block copolymer of $n$-butyl acrylate ( $n$-BA) and an acrylamide monomer bearing triple hydrogen bonding site with the complementary [ $(N-(6-a c-$ etoamidopyridin-2-yl)acrylamide] (DAD) sequence (PBA$b$-PDAD) by the sequential RAFT polymerizations. And, the complexation via specific hydrogen bonding between these block copolymers in $\mathrm{CHCl}_{3}$ provides an unprecedented method for the construction of vesicles (unpublished data). However, systematic study of the self-assembly behavior of PBA- $b$-PDAD has never been undertaken. Actually, Poly(n-butyl acrylate) (PBA) is one of the commonly used hydrophobic polymers. Hydrophilic polymer PDAD is novel both in structure and character, which has pendant triple hydrogen-bonding units. Therefore, it is very interesting to have insight into the self-assembly behavior of this novel block copolymer PBA- $b$-PDAD in aqueous solutions.

Mediators have been widely used in a variety of polymerization. For example, Lewis acid has been used as the mediator for the stereo-structure control in living radical polymerization [27]. A bulky fluoro-alcohols like $\left(\mathrm{CF}_{3}\right)_{3} \mathrm{COH}$ and $\left(\mathrm{CF}_{3}\right)_{2} \mathrm{CHOH}$ have also been reported as the mediators for the syndiospecific radical polymerization of ester-type monomers, such as vinyl acetate. Recently, we have explored the nucleobase-mediated stereospecific radical polymerization. Utilizing the highly soluble 1-octylthymine in $\mathrm{CH}_{2} \mathrm{Cl}_{2}$ at $-78^{\circ} \mathrm{C}$ as mediator, the syndiospecific radical polymerization of complementary acrylamide monomer DAD yielded syndiotactic rich polymers up to $r=84 \%$ [28].

Herein, we report the synthesis of triple hydrogen-bonding block copolymers (PBA- $b$-PDAD) by the RAFT polymerization using 1-octylthymine as the mediator (Fig. 1). We

Key Laboratory of Polymer Ecomaterials, Changchun Institute of Applied Chemistry, Chinese Academy of Sciences, Changchun 130022, China "Corresponding author (email: youhua.tao@ciac.ac.cn) 
also studied the self-assembly behavior of these novel block copolymer PBA-b-PDAD in aqueous solutions (Fig. 2). The chemical and physical properties of these triple hydrogen-bonding block copolymers were studied by ${ }^{1} \mathrm{H}$ NMR spectroscopy, gel permeation chromatography (GPC), transmission electron microscopy (TEM) and dynamic light-scattering (DLS).

\section{EXPERIMENTAL SECTION}

\section{General procedures}

Acrylamide monomer DAD and 1-octylthymine was synthesized as described earlier. Tetrahydrofuran (THF) and toluene was refluxed over $\mathrm{CaH}_{2}$, then distilled, degassed and stored under a nitrogen atmosphere. ${ }^{1} \mathrm{H}$ NMR spectra were recorded on a Varian Unity-400 spectrometer using dimethylsulfoxide as solvent. GPC was used to determine the molar masses and molar mass distributions (polydispersities, PDIs) of the synthesized polymers. DLS measurement was performed on a Wyatt QELS instrument with a vertical polarized He-Ne laser (DAWN EOS, Wyatt Technology, USA). The scattering angle was fixed at $90^{\circ}$. All DLS samples were prepared by using copolymers dissolved in spectrophotometric grade $\mathrm{CHCl}_{3}(1 \% \mathrm{w} / \mathrm{v})$, and the solution was then filtered with $4.5 \mathrm{~mm}$ polytetrafluoroethylene (PTFE) syringe filters. The tapping mode atomic force microscopy (AFM) images were obtained with a multimode scanning probe microscope coupled to a Nanoscope IIIa controller (Digital Instruments/Veeco, Santa Barbara, CA, USA) under ambient conditions by casting dilute solution of PBA- $b$-PDAD aggregates on a mica surface.

\section{Synthesis of triple hydrogen-bonding block copolymers in} the presence of 1-octylthymine

The typical procedure was as follows: macro-CTA (53 mg, $0.005 \mathrm{mmol}$ ), DAD (143 mg, $0.7 \mathrm{mmol}), 1$-octylthymine (250 mg, $1.05 \mathrm{mmol}$ ) and 2,2-azobisisobutyronitrile (AIBN) (0.1 mg, $0.0005 \mathrm{mmol}$ ) were dissolved in $\mathrm{CHCl}_{3}(2$ $\mathrm{mL})$. The reaction was then placed in an oil bath at $60^{\circ} \mathrm{C}$ for $12 \mathrm{~h}$. Then, the polymerization was terminated by cooling the reaction mixture to $-78^{\circ} \mathrm{C}$. The mixture was dialyzed in methanol for 7 days. The white solid were dried in vacuum and characterized by ${ }^{1} \mathrm{H}$ NMR and GPC (polymethyl methacrylate standards).

\section{Formation of PBA- $b$-PDAD micelles}

PBA- $b$-PDAD (0.05 g) was dissolved in $100 \mathrm{~mL}$ of dimethyl formamide (DMF), followed by the filtration of the solution through a $0.45 \mu \mathrm{m}$ Teflon membrane filter (Chromatographic Specialties, Inc.). The micelles were formed by the addition of the resulting DMF solution into water at a rate of one drop per $60 \mathrm{~s}$ with continuous stirring. The micelle solution was stirred for $24 \mathrm{~h}$ and then dialyzed in water using a cellulose dialyzer tube.

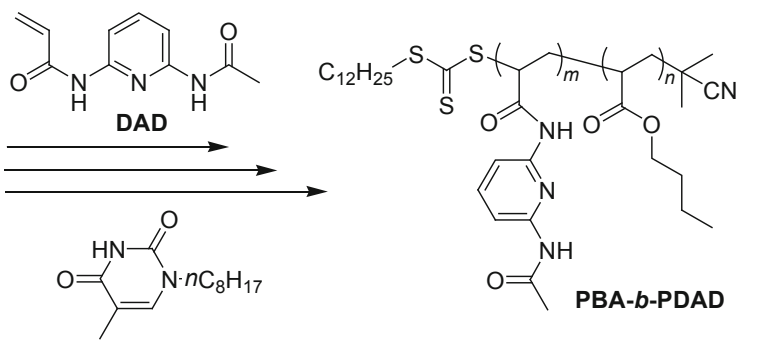

Additive: 1-octylthymine

$60^{\circ} \mathrm{C}, \mathrm{AIBN}, \mathrm{CHCl}_{3}$

Figure 1 Synthesis of the triple hydrogen-bonding-containing diblock copolymers using RAFT polymerization in the presence of 1-octylthymine.

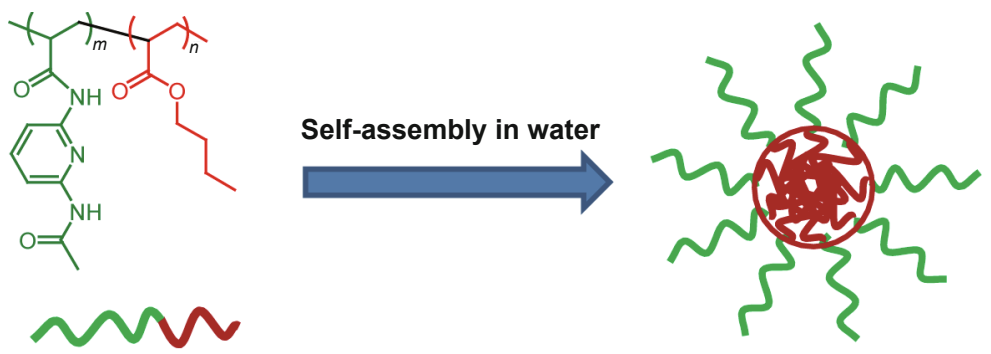

Figure 2 The chemical structure of amphiphilic triple hydrogen-bonding block copolymers PBA- $b$-PDAD and the scheme of the micelles formation. 


\section{RESULTS AND DISCUSSION}

Synthesis of the triple hydrogen-bonding block copolymers in the presence of 1-octylthymine

The synthesis of macro-CTA PBA was reported elsewhere. PBA with different degree of polymerization were prepared to investigate the effect of chain length on the self-assembly behavior of the resultant triple hydrogen-bonding block copolymers. It is well-known that diaminopyridine can form triple hydrogen bonds with thymine or its derivatives [28]. Meanwhile, it has already been demonstrated that this kind of triple hydrogen bonding interaction can be used to control polymer tacticity, to mediate polymer composition or sequence and to template polymerization [28]. Recent reports documented the utility of 1-octylthymine as the mediator in the homo-polymerization of DAD monomer. Here, the synthesis of triple hydrogen-bonding block copolymers in the presence of 1-octylthymine was examined (Fig. 1). The RAFT block copolymerization was carried out in $\mathrm{CHCl}_{3}$, at $60^{\circ} \mathrm{C}$ in the presence of 1-octylthymine as the mediator. The degree of polymerization (DP) was varied by adding different amounts of the monomer. The resultant block copolymers were purified by dialysis in methanol, which removed residual monomers and mediator. The results of the polymerization were listed in Table 1. Satisfyingly, high conversion (>99\%) was achieved for each RAFT block copolymerization. As can be seen in Table 1 , The $M_{\mathrm{n}}$ of the resultant PBA- $b$-PDAD increased with increasing feed ratio, ranging from 24.9 to $31.2 \mathrm{kDa}$. The $M_{\mathrm{n}}$ of the block copolymer could also be calculated from
${ }^{1} \mathrm{H}$ NMR. As can be seen in Table 1 , the $M_{\mathrm{n}}$ of the resultant PBA- $b$-PDAD from GPC was consistent with the ${ }^{1} \mathrm{H}$ NMR results. GPC traces of the synthesized diblock copolymers were summarized in Fig. 3. Though GPC traces of the block copolymers clearly exhibit monodisperse signals, relatively broader PDIs $\left(M_{\mathrm{w}} / M_{\mathrm{n}}>1.7\right)$ were obtained in the presence of 1-octylthymine, which might be attributed to the slightly slower RAFT process in the presence the mediator.

The ${ }^{1} \mathrm{H}$ NMR spectrum of the obtained triple hydrogenbonding block copolymers prepared by RAFT polymerization in the presence of 1-octylthymine is shown in Fig. 4. The characteristic peaks at $\delta=7.57 \mathrm{ppm}$ were assigned to the diaminopyridine group. The characteristic peaks at $\delta=$ $3.93 \mathrm{ppm}$ were attributed to that of the PBA segment of the polymer. FTIR spectra (Fig. 5) analysis of the block polymer showed the absorbance at 3430, 1727 and $1631 \mathrm{~cm}^{-1}$, respectively, which are attributed to the pyridine group, amide bond and ester bond in the block polymer. These results further demonstrated the successful synthesis of triple hydrogen-bonding block copolymers PBA- $b$-PDAD in the presence of mediator.

\section{Self-assembly behavior of PBA- $b$-PDAD in water}

Amphiphilic block copolymers can self-assemble into a wide range of nano-scale structures such as spherical micelles, rods, or vesicles, which are of interest in a number of fields, such as drug delivery, removal of pollutants from water, and stabilization of emulsion polymerization [29]. PBA is one of the commonly used hydrophobic polymers. A number of polymeric micelles formed from PBA-based

Table 1 Synthesis of triple hydrogen-bonding block copolymers in the presence of 1-octylthymine ${ }^{a}$

\begin{tabular}{cccccc}
\hline Polymer & Conv. $(\%)$ & $M_{\mathrm{n}, \mathrm{th}}(\mathrm{kDa})$ & $M_{\mathrm{n}, \mathrm{NMR}}(\mathrm{kDa})^{\mathrm{b}}$ & $M_{\mathrm{n}, \mathrm{GPC}}(\mathrm{kDa})^{\mathrm{c}}$ & $M_{\mathrm{w}} / M_{\mathrm{n}}^{\mathrm{c}}$ \\
\hline $\mathrm{PBA}_{80}-b-\mathrm{PDAD}_{80}$ & $>99$ & 26.8 & 27.0 & 24.9 & 1.8 \\
$\mathrm{PBA}_{80}-b-\mathrm{PDAD}_{120}$ & $>99$ & 34.9 & 35.2 & 30.6 & 1.8 \\
$\mathrm{PBA}_{100}-b-\mathrm{PDAD}_{100}$ & $>99$ & 33.4 & 33.6 & 29.5 & 1.8 \\
$\mathrm{PBA}_{100}-b-\mathrm{PDAD}_{160}$ & $>99$ & 45.6 & 46.0 & 31.2 & 1.8 \\
\hline
\end{tabular}

a) Polymerization in $\mathrm{CHCl}_{3}$ at $60^{\circ} \mathrm{C}$ with $\mathrm{AIBN}$ as the initiator and 1-octylthymine as the additive. b) Determined by ${ }^{1} \mathrm{H}$ NMR. c) Determined by GPC analysis.

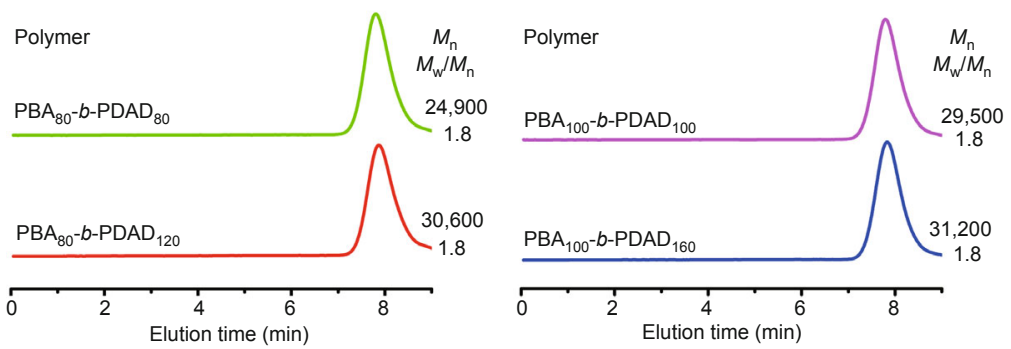

Figure 3 GPC traces of PBA- $b$-PDAD diblock copolymers. 


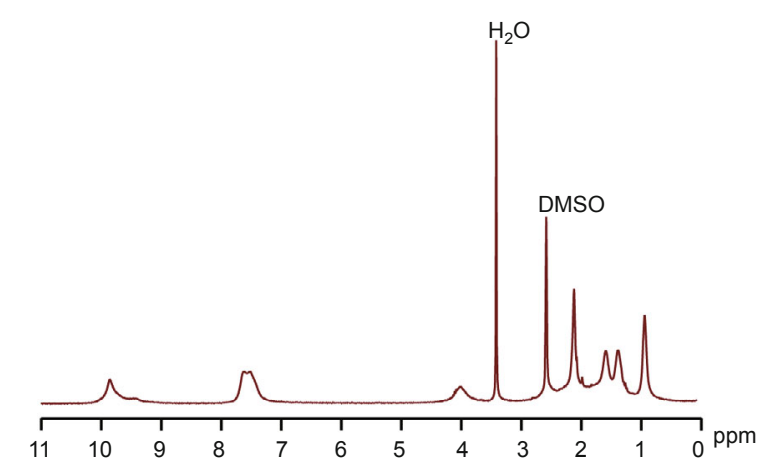

Figure $4{ }^{1} \mathrm{H}$ NMR spectrum (400 $\mathrm{MHz}, \mathrm{CDCl}_{3}$ ) of PBA- $b$-PDAD using RAFT polymerization in the presence of 1-octylthymine.

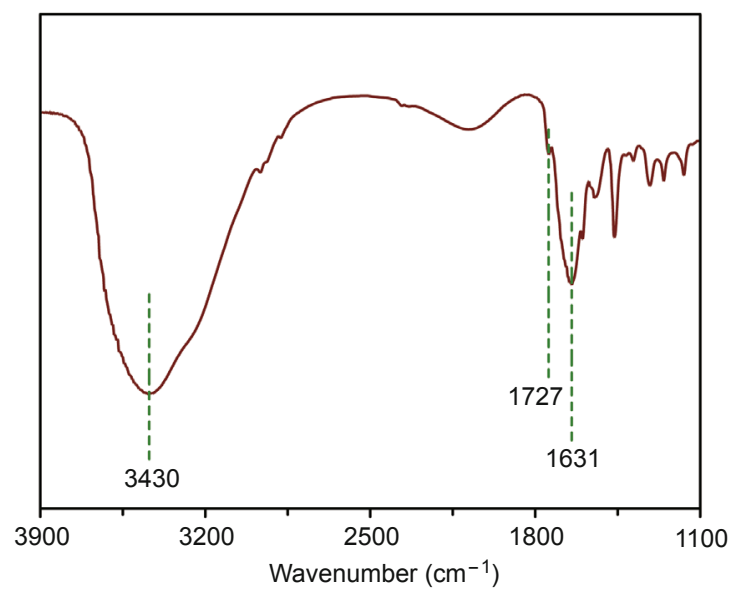

Figure 5 The FTIR spectrum of PBA- $b$-PDAD using RAFT polymerization in the presence of 1-octylthymine.

amphiphilic diblock copolymers have been investigated for various applications. Hydrophilic polymer PDAD is novel both in structure and character, which have pendant triple hydrogen-bonding units. Therefore, it is very interesting to have insight into the self-assembly behavior of this novel triple hydrogen-bonding block copolymer PBA- $b$-PDAD in aqueous solutions. PBA- $b$-PDAD micelles were formed by first dissolving block copolymer in DMF and then gradually adding water as a non-solvent for PBA segments (Fig. 2). The micelle solution was stirred for $24 \mathrm{~h}$ and then dialyzed in water using a cellulose dialyzer tube. Fig. 6 showed the typical TEM image of PBA- $b$-PDAD micelles in water. As shown in the figure, spherical micelles with a narrow size distribution were obtained from amphiphilic triple hydrogen-bonding block copolymer PBA- $b$-PDAD. According to the calculation results from TEM, the size of the micelles is of $\sim 52 \mathrm{~nm}$. The average size and size distribution of the micelles (PDI) could also be determined from DLS measurement (Fig. 7). The hydrodynamic radius was
$53 \mathrm{~nm}$ from DLS method, which was well consistent with the TEM results.

PBA- $b$-PDAD copolymers with different length of hydrophobic and hydrophilic segments were used to prepare micelles of different dimensions. The average size of the micelles was investigated by DLS (Fig. 7). The particle size varied considerably, depending on the length of the PDAD segments, and ranged from 30 to $53 \mathrm{~nm}$ (Fig. 7). This observation was reasonable because a decrease in the PDAD segments length implied that there was a relative increase in the hydrophobic interaction of copolymers, which would certainly lead to tight aggregation of the hydrophobic PBA segments. A similar phenomenon was also observed in other micelle systems. The advantage of triple hydrogen-bonding micelles is that these materials can be used as intelligent drug delivery vehicles. Triple hydrogenbonding can make the drug delivery more intelligent and

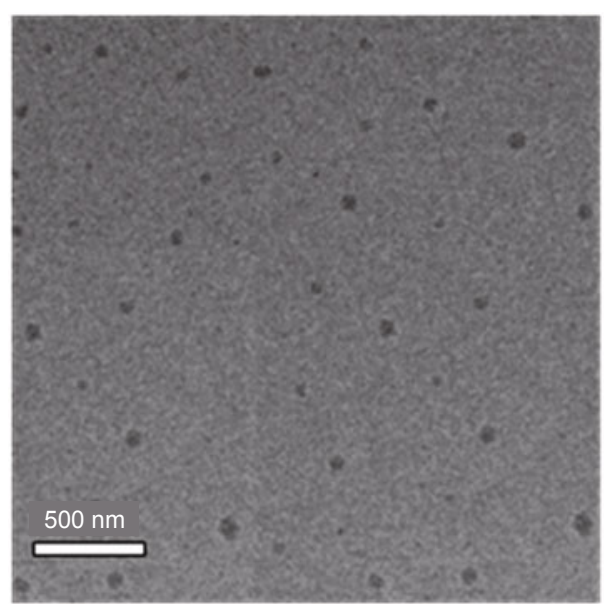

Figure 6 TEM image of $\mathrm{PBA}_{100}-b-\mathrm{PDAD}_{160}$ polymeric micelles in water.

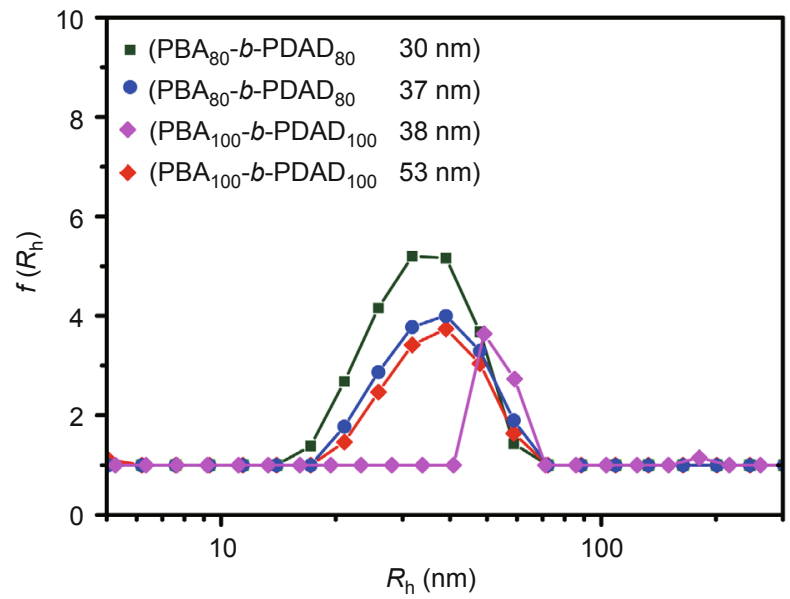

Figure 7 Effect of the length of PDAD segments in copolymer on the hydrodynamic radius $\left(R_{\mathrm{h}}\right)$ of micelles in aqueous solution. 
adapt to the $\mathrm{pH}$ value variation of physiological environment in vivo.

\section{CONCLUSIONS}

In conclusion, sequential RAFT polymerizations were found to be the effective methods for the synthesis of triple hydrogen-bonding block copolymers. The amphiphilic triple hydrogen-bonding block copolymers with $M_{\mathrm{n}}$ up to $24,900 \mathrm{Da}$ and relatively broader molecular weight distribution could be produced in combination with 1-octylthymine as mediator. Micelles formed by these copolymers were directly observed by using TEM and DLS. Relative length of the hydrophobic and hydrophilic segments strongly influenced the hydrodynamic radius of the spherical micelles. Taken together, these PBA- $b$-PDAD micelles with pendant triple hydrogen-bonding groups could be a potential new vehicle for drug delivery.

\section{Received 21 August 2015; accepted 31 August 2015;} published online 14 September 2015

1 Lee SH, Ouchi M, Sawamoto M. Supramolecular X-shaped homopolymers and block polymers by midsegment complementary hydrogen bonds: design of bifunctional initiators with interactive sites for metal-catalyzed living radical polymerization. Macromolecules, 2012, 45: 3702-3710

2 Lo PK, Sleiman HF. Nucleobase-templated polymerization: copying the chain length and polydispersity of living polymers into conjugated polymers. J Am Chem Soc, 2009, 131: 4182-4183

3 Rakotondradany F, Palmer A, Toader V, et al. Hydrogen-bond self-assembly of DNA-analogues into hexameric rosettes. Chem Comm, 2005, 5441-5443

4 McHale R, Patterson JP, Zetterlund PB, et al. Biomimetic radical polymerization via cooperative assembly of segregating templates. Nat Chem, 2012, 4: 491-497

5 Fathalla M, Lawrence CM, Zhang N, Sessler JL, Jayawickramarajah J. Base-pairing mediated non-covalent polymers. Chem Soc Rev, 2009, 38: 1608-1620

6 McHale R, O’Reilly RK. Nucleobase containing synthetic polymers: advancing biomimicry via controlled synthesis and self-assembly. Macromolecules, 2012, 45: 7665-7675

7 Ware T, Hearon K, Lonnecker A, et al. Triple-shape memory polymers based on self-complementary hydrogen bonding. Macromolecules, 2012, 45: 1062-1069

8 Spijker HJ, Delft FL, Hest JCM. Atom transfer radical polymerization of adenine, thymine, cytosine, and guanine nucleobase monomers. Macromolecules, 2007, 40: 12-18

9 South CR, Weck M. Template-enhanced ring-opening metathesis polymerization. Macromolecules, 2007, 40: 1386-1394

10 Wang K, Liu Y, Li C, et al. Cyclodextrin-responsive micelles based on poly(ethylene glycol)-polypeptide hybrid copolymers as drug carriers. ACS Macro Lett, 2013, 2: 201-205

11 Ishihara Y, Bazzi HS, Toader V, Godin F, Sleiman HF. Molecule-responsive block copolymer micelles. Chem A Europ J, 2007, 13: 4560-4570

12 Goh TK, Yamashita S, Satoh K, et al. Highly efficient synthesis of low polydispersity core cross-linked star polymers by Ru-catalyzed living radical polymerization. Macromol Rapid Comm, 2011, 32:
456-461

13 Khan A, Haddleton M, Hannon MJ, Kukulj D, Marsh A. Hydrogen bond template-directed polymerization of protected 5'-acryloylnucleosides. Macromolecules, 1999, 32: 6560-6564

14 Kang Y, Lu A, Ellington A, Geweett MC, O’Reilly RK. Effect of complementary nucleobase interactions on the copolymer composition of RAFT copolymerizations. ACS Macro Lett, 2013, 2: 581-586

15 Drechsler U, Thibault RJ, Rotello VM. Formation of recognition-induced polymersomes using complementary rigid random copolymers. Macromolecules, 2002, 35: 9621-9623

16 Bazzi HS, Bouffard J, Sleiman HF. Self-complementary ABC triblock copolymers via ring-opening metathesis polymerization. Macromolecules, 2003, 36: 7899-7902

17 Binder WH, Kluger C. Combining ring-opening metathesis polymerization (ROMP) with sharpless-type "Click" reactions: an easy method for the preparation of side chain functionalized poly (oxynorbornenes). Macromolecules, 2004, 37: 9321-9330

18 Bazzi HS, Sleiman HF. Adenine-containing block copolymers via ring-opening metathesis polymerization: synthesis and self-assembly into rod morphologies. Macromolecules, 2002, 35: 9617-9620

19 Gibson C, Marshall EL, North M, Robsona DA, Williams PJ. Thymine functionalised polymers via living ring-opening metathesis polymerisation. Chem Comm, 1997, 12: 1095-1096

20 Gareth DR, Gibson VC, Hursthouse MB, et al. Synthesis of nucleic-acid base containing norbornene derivatives as monomers for ring-opening-metathesis-polymerization. J Chem Soc, Perkin Trans 1, 2001, 24: 3365-3381

21 Jennings J, Beija M, Kennon JT, et al. Advantages of block copolymer synthesis by RAFT-controlled dispersion polymerization in supercritical carbon dioxide. Macromolecules, 2013, 46: 6843-6851

22 Zhang K, Fahs GB, Aiba M, Moore RB, Long TE. Nucleobase-functionalized $\mathrm{ABC}$ triblock copolymers: self-assembly of supramolecular architectures. Chem Comm, 2014, 50: 9145-9148

23 Kang Y, Pitto-Barry A, Willcock H, et al. Exploiting nucleobase-containing materials-from monomers to complex morphologies using RAFT dispersion polymerization. Polym Chem, 2015, 6: 106-117

24 Zhang K, Aiba M, Fahs GB, et al. Nucleobase-functionalized acrylic ABA triblock copolymers and supramolecular blends. Polym Chem, 2015, 6: 2434-2444

25 Lutz JF, Thünemann AF, Rurack K. DNA-like 'Melting' of adenineand thymine-functionalized synthetic copolymers. Macromolecules, 2005, 38: 8124-8126

26 Mather BD, Baker MB, Beyer FL, et al. Supramolecular triblock copolymers containing complementary nucleobase molecular recognition. Macromolecules, 2007, 40: 6834-6845

27 Kamigaito M, Satoh K. Stereoregulation in living radical polymerization. Macromolecules, 2008, 41: 269-276

28 Tao Y, Satoh K, Kamigaito M. Nucleobase-mediated stereospecific radical polymerization and combination with RAFT polymerization for simultaneous control of molecular weight and tacticity. Macromol Rapid Comm, 2011, 32: 226-232

29 Tao Y, Yang Y, Shi D, et al. Micelles formation of polystyrene-co-poly ( $\mathrm{N}$-acryloylthymine) and its aggregation behavior induced by triple hydrogen bonding. Polymer, 2012, 53: 1551-1557

Acknowledgements This work was supported by the National Natural Science Foundation of China (21304085).

Author contributions Wang S, Tao Y and Wang X designed the project and the experiments. Wang $\mathrm{S}$ performed the experiments. Wang $\mathrm{S}$ and Tao $\mathrm{Y}$ analyzed the results and wrote the paper.

Conflict of interest The authors declare that they have no conflict of interest. 


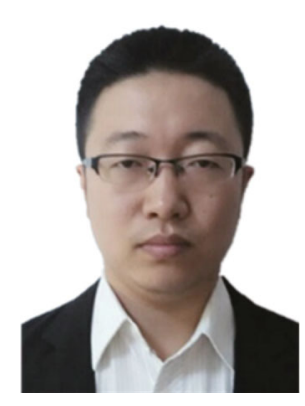

Shixue Wang is a research assistant at the Key Laboratory of Polymer Ecomaterials, Changchun Institute of Applied Chemistry (CIAC), Chinese Academy of Sciences (CAS). He received his MSc and BSc degrees from the Department of Chemistry, Jilin University in 2011 and 2008, respectively. His research interests include design and synthesis of novel monomers, methodology in controlled precision polymerization, and characterization of the properties of new polymers.
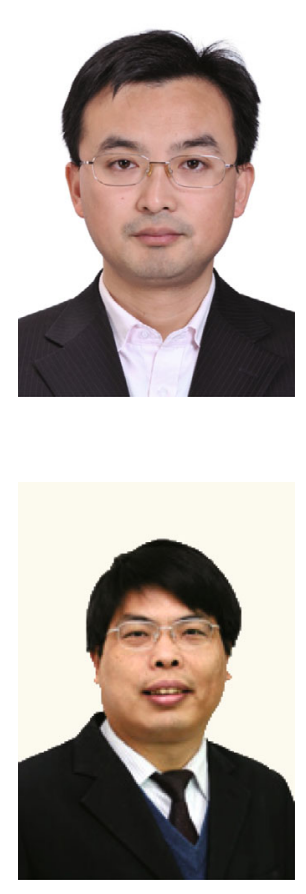

Youhua Tao received his $\mathrm{PhD}$ degree in polymer chemistry from CIAC, CAS under the direction of Prof. Xianhong Wang. After a postdoctoral stay at Nagoya University in Japan, he moved to the University of Colorado at Boulder as a postdoc. In June 2013, he accepted a full professor appointment at CIAC. His current research mainly focuses on the precision synthesis of amino-acid polymers.

Xianhong Wang received his $\mathrm{PhD}$ degree majored in polymer physics and chemistry, in CIAC, CAS. He worked at CIAC since Dec. 1993, and was promoted to full professor in Dec. 1997. His research interests are biodegradable $\mathrm{CO}_{2}$ copolymers and conducting polymers. He has published more than 150 papers, and owned two US patents, one Japanese patent, and 60 Chinese patents.

中文摘要 本文以 1 -辛烷基胸腺嘧啶为调节剂, 通过RAFT聚合合成了含有三重氢键的两亲性嵌段聚合物(PBA- $b$-PDAD). 凝胶渗透色 谱测试表明, PBA- $b-P D A D$ 的数均分子量可达 $24,900 \mathrm{Da}$, 并具有相对较宽的分子量分布. PBA- $b-P D A D$ 可在水溶液中组装成具有核壳结 构的球形胶束. 透射电镜和动态光散射研究表明, 该球形胶束的粒径在 $50 \mathrm{~nm}$ 左右. 此类含三重氢键的球形胶束, 在生物材料以及药物 缓释领域具有潜在的应用价值. 